\section{RADIOGRAPHS AND DISPROPORTION}

BY

\section{J. V. O'SULLIVAN, M.D., F.R.C.S., M.R.C.P., M.A.O., M.R.C.O.G.}

Assistant Obstetric Surgeon, City of London Maternity Hospital and Queen Mary's Hospital for the East End, Surgeon, Emergency Medical Service

\section{WITH AN ADDENDUM}

BY

\section{F. M. CRA WSHAW, M.A., M.B., B.Chir., D.M.R.E. Radıologist, Emergency Medical Service}

Disproportion is one of the most worrying abnormalities in midwifery. Particularly is this so in medium and minor degrees of contracted pelvis, in which diminished reserve capacity of the pelvis is so difficult to assess. Marked overlap, of course, renders the diagnosis easy. It is in the type of case usually treated by a test labour that radiographs are of the greatest importance. The method employed in this series of cases is simple, and can be carried out by any radiologist using the ordinary apparatus.

\section{A New Method of Estimating Disproportion}

Dr. J. W. McLaren, radiologist to Queen Mary's Hospital for the East End, first drew my attention to a method which I was soon convinced was of great value as a visual aid in test labour cases. I conduct no test labour now without taking special films of the case. At the outbreak of war the Lyle Maternity Wing of Queen Mary's Hospital was closed and I continued the method at the Kingston County Hospital, where Dr. Crawshaw, the E.M.S. radiologist, has given every co-operation and has further developed the method. All cases analysed in this paper were cases of disproportion. The diagonal conjugates varied between $3 \frac{3}{4}$ and $4 \frac{1}{2}$ inches, the great majority lying between 4 and $4 \frac{1}{4}$ inches. The foetal heads in all cases remained above the brim at ante-natal visits and could not be " pushed in" by the usual manœuvre of pressure downwards and backwards into the pelvis or by sitting the patient up. Overlap was present in several cases, as shown by ordinary palpation and by Munro Kerr's method. All these women were examined weekly towards the end of pregnancy, and on each occasion attempts to make the head engage failed.

I would like to add one clinical finding most helpful in giving an opinion; it is that in those cases in which the position remained constant-i.e., L.O.A. or R.O.A. at the last three or four visits-the patients delivered themselves spontaneously.

Patients were radiographed in the last two weeks of pregnancy, and the usual method of calculating the expectant date (adding seven days to the L.M.P. and going forward nine months) was adopted. Some of the radiographs were taken when the patient was actually in the first stage of labour. Dr. Crawshaw gives in the Addendum the technical details of the method used in taking the radiographs. I would like, however, to emphasize that the antero-posterior film should be large enough to show the whole foetus and the brim of the pelvis. This will enable the radiologist to exclude abnormalities in the foetus; also to show the lie, position, presentation, and degree of flexion of the head, and the relation of the head to the conjugate diameter of the brim. If the head lies centrally the anteroposterior diameter of the brim and of the head are magnified proportionately when a true lateral film is taken. If the head is lying obliquely at the brim an oblique diameter is of course projected, and this diameter is always greater than the biparietal.

\section{Interpreting the Films}

It will be remembered that the foetus during its progress through the pelvis traverses the obstetrical pelvic axis. This axis at its commencement corresponds with the first part of the curve of Carus and may be represented at the brim by a line drawn upwards at right angles to the mid-point of the plane of the brim. Making use of this knowledge, I found the best way to read the lateral radiographs was to draw a line on the films between the promontory of the sacrum and the upper posterior margin of the symphysis pubis, and from each extremity of this antero-posterior diameter of the brim to erect perpendicular lines, thus forming the sides of a "box" (see diagram). This plan has

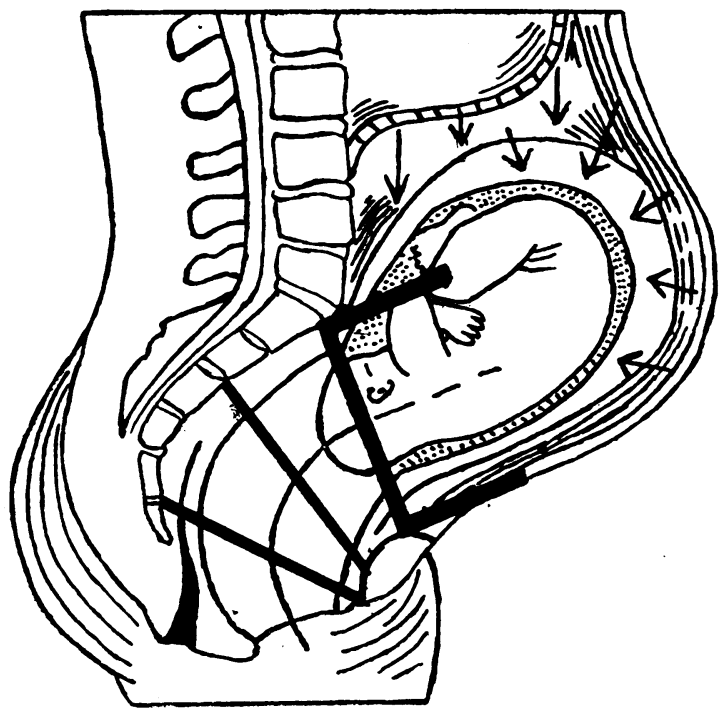

Diagram showing perpendicular lines erected at extremities of true conjugate to form sides of box.

been followed in the reading of all the films, and wherever the foetal head fitted easily between the perpendicular lines it could be safely assumed that it would go through the brim. The erection of these perpendicular lines makes the reading of the films simple and accurate. It is self-evident that if the biparietal diameter does not fit into the "box" no other diameter can, and Caesarean section is advisable.

In our opinion a good lateral film is the radiological counterpart of Munro Kerr's clinical method. Originally we took the lateral films with the patient lying on her side on the $x$-ray table, being careful that the thighs were not flexed sufficiently to obscure the top of the symphysis. Recently, however, Dr. Crawshaw has taken the lateral view with the patient standing up and leaning forward with her hands grasping the back of a chair. It will be recalled that this is the natural position for a patient to assume when she is seized with a pain while walking about during the first stage of labour. This position brings the uterus, and with it the foetus, at right angles to the "position of entry."

\section{Results}

I have analysed 102 cases over the last three years and find that the radiologist's report was wrong in only six instances. In three of these the report said the head would not pass, and it moulded through, with the aid of forceps, in one case ; in one the report said the head would pass, and it did not; while in the remaining two the report said the heads might pass, and Caesarean section was necessary. 
I have had cases in which, though the head could not be pushed in clinically, the film showed that it would pass. In one case I was so doubtful that a week after the expectant date I took the patient to the theatre for examination under anaesthesia and probably for Caesarean section. Under the anaesthetic the head would partially enter the brim. In view of this and the definite radiological report I ruptured the membranes with a Drew Smythe catheter, and spontaneous delivery of a living child followed during the next twenty-four hours. There were other very doubtful cases in which this simple radiological aid was of the greatest help to me.

I am convinced that this method is of great value in cases of disproportion. The films should be taken as near the expectant date as possible if the fullest aid is to be obtained from them.

\section{ADDENDUM}

The method of estimating disproportion described in this paper depends upon the fact that if any diameter of the head and the true conjugate of the pelvis are equidistant from the film the magnification of these is the same. Hence it can only be of use in vertex presentations with the head central in the pelvis. It gives no information in any other presentation or in cases of outlet contraction. In spite of these limitations it is of considerable value to the obstetrician, because it is in the true conjugate diameter that disproportion is most often encountered.

Examination of the antero-posterior and lateral views of the pelvis enables the radiologist to see if the head is lying transversely or obliquely: as already stated, if the foetal head lies obliquely in the pelvis a diameter larger than the biparietal is projected. In a high percentage of cases clinically suspected of disproportion the lateral radiograph shows that there is a large clearance between the size of the foetal skull and the true conjugate, and disproportion can be excluded. The question of what relation between the foetal skull and the true conjugate constitutes disproportion is a real difficulty. Mere direct comparison of the size of the bony parts is clearly not sufficient, because there are always unknown factors-e.g., the degree of moulding and the thickness of the soft parts-to be considered. The radiologist must therefore make his rules of interpretation from experience of what happens in labour.

In the above series of cases the rule has been used that where the biparietal diameter is greater than the true conjugate disproportion exists; where it is equal to the true conjugate the head will only go through with moulding; and where it is less than the true conjugate no difficulty from disproportion need be anticipated. It may be that this rule will have to be altered as a result of further experience, but enough has been learnt to show that it is not far from the truth. In those cases in which the head is lying obliquely and in which the oblique diameter of the skull projected is greater than the true conjugate it is not possible to be certain whether disproportion exists or not.

As to the actual radiography, the lateral films may be taken with the patient lying on her side or, if a vertical Potter-Bucky is available, standing with the arms supported on the back of a chair. The hips should not be flexed, as this may cause the shadow of the symphysis to be hidden by the shadows of the femora. To be of use the film should not be far from a true lateral as judged by the superimposition of the shadows of the femoral heads.

Our grateful thanks are due to Dr. McLaren (who would have collaborated with us but for his absence on active service). Our thanks are also due to Mr. Kidd, superintendent of Kingston County Hospital; to Mr. A. McAlister of Queen Mary's Hospital, Stratford; and to the resident and the radiological staffs of the two hospitals.

\section{SULPHANILAMIDE IN LOCAL TREATMENT OF SKIN INFECTIONS}

\author{
BY
}

\author{
A. G. MARShALL, M.B., B.Chir. \\ Flight Lieutenant, R.A.F.V.R.
}

Localized infections of the skin such as sycosis and impetigo contagiosa have been found difficult to heal, especially when established for some time. In the Services, although these conditions are not common, the time factor and the prevention of cross-infection combine to make the problem an important one. Older methods of treatment, such as the application of ointments after bathing the lesions, are tedious and in many cases ineffective. Gentian-violet paint was not used in the following series of cases. The recent satisfactory reports of the treatment of wounds by the local application of sulphanilamide powder suggested the extension of this method to such conditions. The results have been most gratifying.

The usefulness of sulphanilamide therapy in skin diseases has been the subject of much discussion, and its value is stated by Smith (1940) to be far from understood. He reports good results in the local application of solutions and ointments to ulcers and infected burns and refers to the advantages of economy and atoxicity. Jaeger (1936) and Merz (1937) have treated skin lesions with the drugs given orally, and locally as solutions in water and glycerin: the latter applied his methods to impetigo. Buchanan (1940) points out the value of crushed tablets of sulphapyridine as a dressing for a pneumococcal ulcer, a treatment which is often of use in varicose ulceration. Under present war conditions I have been able to trace only one author who specifically mentions the use of the powder for infections of the skin : MacKenna (1940) employed it under a dressing of zinc paste for impetigo, and stated that it is often successful.

Method
The patients were treated in hospital as ambulant but isolated cases. The scabs were bathed off three or four times daily with a warm $2 \%$ solution of sodium bicarbonate. The sulphanilamide powder (streptocide) was applied direct to the raw surface, where it sticks well, and was left with no dressing. The treatment was usually carried out by the patient after the first few times, and he used more powder when required. It was of course essential to continue regular shaving in the healthy areas, and the hair was clipped short elsewhere. Frequent shampoos were given. In cases with thick masses of exudate which did not separate easily a gauze pad soaked in liquid paraffin was applied for twelve hours, and the treatment then continued, the old exudate separating readily with this application. Other oily dressings were rigidly avoided, as they were found to spread the infection. In certain cases, described below, ascorbic acid was prescribed by mouth, starting with $500 \mathrm{mg}$. daily, the dose, after saturation, being reduced progressively to $50 \mathrm{mg}$. daily.

\section{Results}

All the cases had already been treated with bathing and ointments. There was immediate and decided improvement after the application of sulphanilamide powder. The surrounding inflammation and the exudation rapidly decreased, and in three days the lesions were sterile and remained so. Case 4 (see below) was at first treated with sulphanilamide ointment, but after a week the lesions had not become sterile nor apparently had they improved. It is therefore suggested that a high concentration is necessary to destroy the staphylococcus. This is unlikely to 